\title{
Tsunami run-up heights of the 2003 Tokachi-oki earthquake
}

\author{
Yuichiro Tanioka ${ }^{1}$, Yuichi Nishimura ${ }^{1}$, Kazuomi Hirakawa $^{2}$, Fumihiko Imamura $^{3}$, Ikuo Abe ${ }^{3}$, Yoshi Abe ${ }^{3}$, Kazuya Shindou $^{3}$, \\ Hideo Matsutomi ${ }^{4}$, Tomoyuki Takahashi ${ }^{4}$, Kentaro Imai ${ }^{4}$, Kenji Harada ${ }^{5}$, Yuichi Namegawa ${ }^{6}$, Yohei Hasegawa ${ }^{7}$, \\ Yutaka Hayashi ${ }^{7}$, Futoshi Nanayama ${ }^{8}$, Takanobu Kamataki ${ }^{8}$, Yoshiaki Kawata ${ }^{9}$, Yoshinobu Fukasawa ${ }^{9}$, \\ Shunichi Koshimura ${ }^{9}$, Yasunori Hada ${ }^{9}$, Yusuke Azumai ${ }^{9}$, Kenji Hirata ${ }^{10}$, Akiyasu Kamikawa ${ }^{11}$, \\ Akifumi Yoshikawa ${ }^{11}$, Toru Shiga ${ }^{11}$, Masaki Kobayashi ${ }^{11}$, and Seiichi Masaka ${ }^{12}$ \\ ${ }^{1}$ Institute of Seismology and Volcanology, Hokkaido University, N10W8 Kita-ku, Sapporo 060-0810, Japan \\ ${ }^{2}$ Graduate School of Environmental Earth Science, Hokkaido University, N10W5 Kita-ku, Sapporo 060-0810, Japan \\ ${ }^{3}$ Disaster Control Research Center, Graduate School of Engineering, Tohoku University, Aoba 06, Sendai 980-8579, Japan \\ ${ }^{4}$ Department of Civil and Environmental Engineering, Faculty of Engineering and Resource Science, \\ Akita University, 1-1 Tegatagakuen, Akita 010-8502, Japan \\ ${ }^{5}$ Disaster Prevention Research Institute, Kyoto University, Gokasho, Uji, Kyoto 611-0011, Japan \\ ${ }^{6}$ Earthquake Research Institute, University of Tokyo, 1-1-1 Yayoi, Bunkyo-ku, Tokyo 113-0032, Japan \\ ${ }^{7}$ Seismology and Volcanology Research Department, Meteorological Research Institute, 1-1 Nagamine, Tsukuba 305-0052, Japan \\ ${ }^{8}$ National Institute of Advanced Industrial Science and Technology, Site C7 1-1-1 Higashi, Tsukuba 305-8567, Japan \\ ${ }^{9}$ Disaster Reduction and Human Renovation Institution, 1-5-2 Wakinohama kaigan-dori, Chuo-ku, Kobe 651-0073, Japan \\ ${ }^{10}$ Deep Sea Research Department, Japan Marine Science and Technology Center, 2-15 Natsuhima-cho, Yokosuka 237-0061, Japan \\ ${ }^{11}$ Sapporo District Meteorological Observatory, N2W18, Cyuou-ku, Sapporo 060-0002, Japan \\ ${ }^{12}$ Japan Meteorological Agency, 1-3-4 Otemachi, Chiyoda-ku, Tokyo 100-8122, Japan
}

(Received November 28, 2003; Revised March 2, 2004; Accepted March 2, 2004)

\begin{abstract}
Tsunami height survey was conducted immediately after the 2003 Tokachi-oki earthquake. Results of the survey show that the largest tsunami height was $4 \mathrm{~m}$ to the east of Cape Erimo, around Bansei-onsen, and locally at Mabiro. The results also show that the tsunami height distribution of the 2003 Tokachi-oki earthquake is clearly different from that of the 1952 Tokachi-oki earthquake, suggesting the different source areas of the 1952 and 2003 Tokachioki earthquakes. Numerical simulation of tsunami is carried out using the slip distribution estimated by Yamanaka and Kikuchi (2003). The overall pattern of the observed tsunami height distribution along the coast is explained by the computed ones although the observed tsunami heights are slightly smaller. Large later phase observed at the tide gauge in Urakawa is the edge wave propagating from Cape Erimo along the west coast of the Hidaka area.
\end{abstract}

Key words: The 2003 Tokachi-oki earthquake, tsunami height survey, tsunami numerical simulation, edge wave.

\section{Introduction}

On 26 September 2003, a large Tokachi-oki earthquake occurred at the southern coast of Hokkaido, Japan. The Japan Meteorological Agency (JMA) estimated the source parameters as follows: origin time, 4:50:07.5 (JST); epicenter, $41^{\circ} 46.7^{\prime} \mathrm{N}, 144^{\circ} 04.7^{\prime} \mathrm{E}$; depth $42 \mathrm{~km}$; the JMA magnitude, $\mathrm{M}_{J}$ 8.0. The seismic moment estimated from the teleseismic body waves was $1.0 \times 10^{21} \mathrm{Nm}$, or $\mathrm{Mw} 8.0$ (Yamanaka and Kikuchi, 2003). The aftershock area was almost the same as that of the 1952 Tokachi-oki earthquake (Ichiyanagi et al., 2004). The earthquake generated large tsunamis causing damage along the southern coast of Hokkaido. Immediately after the earthquake, the tsunami survey was conducted by tsunami researchers from all over Japan. Several survey teams covered all the coasts of the eastern Hokkaido and the northern Tohoku regions. The tsunami was also observed at the tide gauges in the Hokkaido and Tohoku regions (Hirata et al., 2003).

In this paper, observed tsunami run-up heights of the 2003

Copy right(c) The Society of Geomagnetism and Earth, Planetary and Space Science (SGEPSS); The Seismological Society of Japan; The Volcanological Society of Japan; The Geodetic Society of Japan; The Japanese Society for Planetary Sciences; TERRAPUB
Tokachi-oki earthquake are compiled and compared with those of the 1952 great Tokachi-oki earthquake. The result of a numerical simulation of the 2003 tsunami is compared with the observed tsunami run-up heights. The generation mechanism of the large later phase observed at the tide gauge in Urakawa is also discussed.

\section{Tsunami Height Survey}

The tsunami assessment was conducted immediately after the earthquake. The survey along the southern coast of Hokkaido and the eastern coast of Tohoku was almost completed by 29th of September, three days after the earthquake. The survey was continued by 2 nd of October and an additional assessment was conducted by JMA on 10th and 11th of October. The quick survey is particularly important for a moderate tsunami of which the run-up heights are less than $3 \mathrm{~m}$. Most of tsunami deposits for such a moderate tsunami exist in a surf zone, so those deposits were quickly disturbed by high waves.

The results of the tsunami height survey are shown in Fig. 1 and Table 1. The tsunami heights are measured from various objects that the tsunami left behind (Fig. 2(a)), the line marked around the buildings by the tsunami (Fig. 2(b)), or eyewitness accounts. Most of tsunami heights are origi- 
Table 1. Tsunami height survey results.

\begin{tabular}{|c|c|c|c|c|c|c|c|}
\hline survey point & $\begin{array}{l}\text { latitude } \\
(\mathrm{N})\end{array}$ & $\begin{array}{l}\text { longitude } \\
\text { (E) }\end{array}$ & $\begin{array}{c}\text { measured } \\
\text { height } \\
\text { (m) }\end{array}$ & $\begin{array}{c}\text { tsunami } \\
\text { arrival } \\
\text { time }\end{array}$ & $\begin{array}{c}\text { tidal } \\
\text { correction } \\
\text { (m) }\end{array}$ & $\begin{array}{l}\text { reference } \\
\text { tide } \\
\text { gauge }^{2)}\end{array}$ & $\begin{array}{l}\text { corrected } \\
\text { height } \\
\text { (m) }\end{array}$ \\
\hline Mukawa & $42^{\circ} 32^{\prime} 39^{\prime \prime}$ & $141^{\circ} 56^{\prime} 45^{\prime \prime}$ & 0.48 & $6: 40$ & 0.70 & Ura & 1.2 \\
\hline Monnbetsu & $42^{\prime \prime} 28^{\prime} 24^{\prime \prime}$ & $142^{\circ} 04^{\prime} 29^{\prime \prime}$ & 0.60 & $6: 40$ & 1.00 & Ura & 1.6 \\
\hline Shizunaigawa & $42^{\prime \prime} 19^{\prime} 50^{\prime \prime}$ & $142^{\circ} 22^{\prime} 04^{\prime \prime}$ & 1.15 & $6: 40$ & -0.05 & Ura & 1.1 \\
\hline Shizunaigawa & $42^{\prime \prime} 19^{\prime} 50^{\prime \prime}$ & $142^{\circ} 22^{\prime} 04^{\prime \prime}$ & 0.84 & $6: 40$ & -0.05 & Ura & 0.8 \\
\hline Irifune & $42^{\prime \prime} 19^{\prime} 31^{\prime \prime}$ & $142^{\circ} 22^{\prime} 14^{\prime \prime}$ & 1.02 & $6: 40$ & -0.15 & Ura & 0.9 \\
\hline Irifune & $42^{\prime \prime} 19^{\prime} 31^{\prime \prime}$ & $142^{\circ} 22^{\prime} 14^{\prime \prime}$ & 1.27 & $6: 40$ & -0.15 & Ura & 1.1 \\
\hline Higashishizunai & $42^{\circ} 18^{\prime} 00^{\prime \prime}$ & $142^{\circ} 27^{\prime} 04^{\prime \prime}$ & 1.79 & $6: 40$ & -0.15 & Ura & 1.6 \\
\hline Mitsuishigawa & $42^{\circ} 14^{\prime} 43^{\prime \prime}$ & $142^{\circ} 34^{\prime} 03^{\prime \prime}$ & 2.24 & $6: 30$ & -0.25 & Ura & 2.0 \\
\hline Mitsuishi & $42^{\circ} 13^{\prime} 21^{\prime \prime}$ & $142^{\circ} 37^{\prime} 42^{\prime \prime}$ & 2.06 & $6: 30$ & -0.20 & Ura & 1.9 \\
\hline Ogifushi & $42^{\circ} 11^{\prime} 32^{\prime \prime}$ & $142^{\circ} 40^{\prime} 58^{\prime \prime}$ & 2.48 & $6: 20$ & -0.20 & Ura & 2.3 \\
\hline Ebuegawa & $42^{\circ} 11^{\prime} 16^{\prime \prime}$ & $142^{\circ} 42^{\prime} 54^{\prime \prime}$ & 2.98 & $6: 20$ & -0.15 & Ura & 2.8 \\
\hline Hidakahorobetsu & $42^{\circ} 11^{\prime} 06^{\prime \prime}$ & $142^{\circ} 43^{\prime} 15^{\prime \prime}$ & 2.15 & $6: 20$ & 0.30 & Ura & 2.5 \\
\hline Urakawa-ko & $42^{\circ} 09^{\prime} 53^{\prime \prime}$ & $142^{\circ} 46^{\prime} 20^{\prime \prime}$ & 1.40 & $6: 20$ & 0.00 & Ura & 1.4 \\
\hline Samani & $42^{\circ} 07^{\prime} 58^{\prime \prime}$ & $142^{\circ} 54^{\prime} 26^{\prime \prime}$ & 2.57 & $6: 00$ & 0.35 & Ura, Uta & 2.9 \\
\hline Samani & $42^{\circ} 07^{\prime} 45^{\prime \prime}$ & $142^{\circ} 54^{\prime} 27^{\prime \prime}$ & 2.80 & $6: 00$ & -0.40 & Ura, Uta & 2.4 \\
\hline Samani & $42^{\circ} 07^{\prime} 53^{\prime \prime}$ & $142^{\circ} 54^{\prime} 40^{\prime \prime}$ & 1.80 & $6: 00$ & 0.50 & Ura, Uta & 2.3 \\
\hline Horoman & $42^{\circ} 05^{\prime} 02^{\prime \prime}$ & $143^{\circ} 02^{\prime} 24^{\prime \prime}$ & 1.28 & $6: 00$ & 0.40 & Ura, Uta & 1.7 \\
\hline Asahi-gyoko & $42^{\circ} 03^{\prime} 49^{\prime \prime}$ & $143^{\circ} 03^{\prime} 54^{\prime \prime}$ & 2.05 & $6: 00$ & 0.20 & Ura, Uta & 2.3 \\
\hline Fuemai-gyoko & $42^{\circ} 02^{\prime} 46^{\prime \prime}$ & $143^{\circ} 06^{\prime} 06^{\prime \prime}$ & 1.67 & $5: 50$ & 0.20 & Uta & 1.9 \\
\hline Fuemai-gyoko & $42^{\circ} 02^{\prime} 37^{\prime \prime}$ & $143^{\circ} 06^{\prime} 10^{\prime \prime}$ & 1.60 & $5: 50$ & 0.30 & Uta & 1.9 \\
\hline Erimo-ko & $42^{\circ} 01^{\prime} 07^{\prime \prime}$ & $143^{\circ} 08^{\prime} 35^{\prime \prime}$ & 2.55 & $5: 40$ & 0.25 & Uta & 2.8 \\
\hline Utabetsu & $41^{\circ} 59^{\prime} 34^{\prime \prime}$ & $143^{\circ} 09^{\prime} 41^{\prime \prime}$ & 0.60 & $5: 40$ & 0.20 & Uta & 0.8 \\
\hline Utabetsuhigashi & $41^{\circ} 59^{\prime} 37^{\prime \prime}$ & $143^{\circ} 09^{\prime} 42^{\prime \prime}$ & 0.95 & $5: 40$ & 0.10 & Uta & 1.1 \\
\hline Utabetsuhigashi & $41^{\circ} 58^{\prime} 57^{\prime \prime}$ & $143^{\circ} 09^{\prime} 57^{\prime \prime}$ & 1.49 & $5: 40$ & 0.30 & Uta & 1.8 \\
\hline Erimotoyo & $41^{\circ} 57^{\prime} 11^{\prime \prime}$ & $143^{\circ} 12^{\prime} 38^{\prime \prime}$ & 2.19 & $5: 20$ & -0.45 & Uta & 1.7 \\
\hline Erimotoyo & $41^{\circ} 57^{\prime} 01^{\prime \prime}$ & $143^{\circ} 13^{\prime} 07^{\prime \prime}$ & 0.70 & $5: 20$ & 0.50 & Uta & 1.2 \\
\hline Cape Erimo & $41^{\circ} 55^{\prime} 23^{\prime \prime}$ & $143^{\circ} 15^{\prime} 08^{\prime \prime}$ & 2.70 & $5: 20$ & 0.50 & Uta & 3.2 \\
\hline Erimo-gyoko & $41^{\circ} 56^{\prime} 00^{\prime \prime}$ & $143^{\circ} 14^{\prime} 37^{\prime \prime}$ & 3.30 & $5: 20$ & -0.10 & Uta & 3.2 \\
\hline Erimo-gyoko & $41^{\circ} 56^{\prime} 07^{\prime \prime}$ & $143^{\circ} 14^{\prime} 39^{\prime \prime}$ & 2.05 & $5: 20$ & -0.05 & Uta & 2.0 \\
\hline Erimo-gyoko & $41^{\circ} 56^{\prime} 09^{\prime \prime}$ & $143^{\circ} 14^{\prime} 39^{\prime \prime}$ & 1.40 & $5: 20$ & 0.50 & Uta & 1.9 \\
\hline Hyakuninhama & $41^{\circ} 56^{\prime} 51^{\prime \prime}$ & $143^{\circ} 14^{\prime} 24^{\prime \prime}$ & 2.55 & $5: 20$ & 0.05 & Uta & 2.6 \\
\hline Hyakuninhama & $41^{\circ} 56^{\prime} 47^{\prime \prime}$ & $143^{\circ} 14^{\prime} 24^{\prime \prime}$ & 2.40 & $5: 20$ & 0.50 & Uta, Hir & 2.9 \\
\hline Hyakuninhama & $41^{\circ} 57^{\prime} 34^{\prime \prime}$ & $143^{\circ} 14^{\prime} 33^{\prime \prime}$ & 3.10 & $5: 20$ & 0.45 & Uta, Hir & 3.6 \\
\hline Hyakuninhama & $41^{\circ} 57^{\prime} 34^{\prime \prime}$ & $143^{\circ} 14^{\prime} 33^{\prime \prime}$ & 3.90 & $5: 20$ & -0.05 & Uta, Hir & 3.9 \\
\hline Hyakuninhama & $41^{\circ} 57^{\prime} 36^{\prime \prime}$ & $143^{\circ} 14^{\prime} 34^{\prime \prime}$ & 4.01 & $5: 20$ & -0.15 & Uta, Hir & 3.9 \\
\hline Hyakuninhama & $41^{\circ} 59^{\prime} 47^{\prime \prime}$ & $143^{\circ} 15^{\prime} 35^{\prime \prime}$ & 3.04 & $5: 20$ & 0.40 & Uta, Hir & 3.4 \\
\hline Hyakuninhama & $41^{\circ} 58^{\prime} 06^{\prime \prime}$ & $143^{\circ} 14^{\prime} 38^{\prime \prime}$ & 3.45 & $5: 20$ & 0.35 & Uta, Hir & 3.8 \\
\hline Hyakuninhama & $41^{\circ} 59^{\prime} 45^{\prime \prime}$ & $143^{\circ} 15^{\prime} 16^{\prime \prime}$ & 3.20 & $5: 20$ & 0.00 & Uta, Hir & 3.2 \\
\hline Hyakuninhama & $41^{\circ} 59^{\prime} 52^{\prime \prime}$ & $143^{\circ} 15^{\prime} 17^{\prime \prime}$ & 2.55 & $5: 20$ & 0.00 & Uta, Hir & 2.6 \\
\hline Hyakuninhama & $42^{\circ} 01^{\prime} 04^{\prime \prime}$ & $143^{\circ} 16^{\prime} 36^{\prime \prime}$ & 2.95 & $5: 20$ & 0.15 & Uta, Hir & 3.1 \\
\hline Shoya-gyoko & $42^{\circ} 02^{\prime} 35^{\prime \prime}$ & $143^{\circ} 17^{\prime} 45^{\prime \prime}$ & 1.90 & $5: 20$ & 0.30 & Hir & 2.2 \\
\hline Shoya-gyoko & $42^{\circ} 02^{\prime} 39^{\prime \prime}$ & $143^{\circ} 17^{\prime} 52^{\prime \prime}$ & 1.50 & $5: 20$ & 0.40 & Hir & 1.9 \\
\hline Shoya & $42^{\circ} 02^{\prime} 49^{\prime \prime}$ & $143^{\circ} 18^{\prime} 45^{\prime \prime}$ & 2.35 & $5: 20$ & 0.35 & Hir & 2.7 \\
\hline Meguro & $42^{\circ} 07^{\prime} 20^{\prime \prime}$ & $143^{\circ} 19^{\prime} 15^{\prime \prime}$ & 1.95 & $5: 20$ & 0.45 & Hir & 2.4 \\
\hline Oshirabetsu & $42^{\circ} 13^{\prime} 29^{\prime \prime}$ & $143^{\circ} 19^{\prime} 07^{\prime \prime}$ & 2.01 & $5: 20$ & 0.45 & Hir & 2.5 \\
\hline Oshirabetsu & $42^{\circ} 13^{\prime} 28^{\prime \prime}$ & $143^{\circ} 19^{\prime} 11^{\prime \prime}$ & 2.59 & $5: 20$ & 0.30 & Hir & 2.9 \\
\hline Hiroo-gawa & $42^{\circ} 16^{\prime} 48^{\prime \prime}$ & $143^{\circ} 19^{\prime} 04^{\prime \prime}$ & 2.70 & $5: 20$ & 0.45 & Hir & 3.2 \\
\hline Hiroo & $42^{\circ} 17^{\prime} 26^{\prime \prime}$ & $143^{\circ} 19^{\prime} 05^{\prime \prime}$ & 1.90 & $5: 20$ & 0.40 & Hir & 2.3 \\
\hline Hiroo & $42^{\circ} 17^{\prime} 22^{\prime \prime}$ & $143^{\circ} 19^{\prime} 17^{\prime \prime}$ & 2.27 & $5: 20$ & 0.40 & Hir & 2.7 \\
\hline Hiroo & $42^{\circ} 17^{\prime} 22^{\prime \prime}$ & $143^{\circ} 19^{\prime} 12^{\prime \prime}$ & 3.43 & $5: 20$ & -0.40 & Hir & 3.0 \\
\hline Hiroo & $42^{\circ} 17^{\prime} 22^{\prime \prime}$ & $143^{\circ} 19^{\prime} 12^{\prime \prime}$ & 3.50 & $5: 20$ & -0.40 & Hir & 3.1 \\
\hline Hiroo & $42^{\circ} 17^{\prime} 22^{\prime \prime}$ & $143^{\circ} 19^{\prime} 12^{\prime \prime}$ & 2.84 & $5: 20$ & -0.40 & Hir & 2.4 \\
\hline Hiroo & $42^{\circ} 17^{\prime} 22^{\prime \prime}$ & $143^{\circ} 19^{\prime} 12^{\prime \prime}$ & 3.10 & $5: 20$ & -0.40 & Hir & 2.7 \\
\hline Hiroo & $42^{\circ} 17^{\prime} 22^{\prime \prime}$ & $143^{\circ} 19^{\prime} 12^{\prime \prime}$ & 2.99 & $5: 20$ & -0.40 & Hir & 2.6 \\
\hline Hiroo & $42^{\circ} 17^{\prime} 22^{\prime \prime}$ & $143^{\circ} 19^{\prime} 12^{\prime \prime}$ & 3.07 & $5: 20$ & -0.40 & Hir & 2.7 \\
\hline Hiroo & $42^{\circ} 17^{\prime} 22^{\prime \prime}$ & $143^{\circ} 19^{\prime} 12^{\prime \prime}$ & 3.12 & $5: 20$ & -0.45 & Hir & 2.7 \\
\hline Hiroo & $42^{\circ} 17^{\prime} 22^{\prime \prime}$ & $143^{\circ} 19^{\prime} 12^{\prime \prime}$ & 3.20 & $5: 20$ & -0.45 & Hir & 2.8 \\
\hline Hiroo & $42^{\circ} 17^{\prime} 22^{\prime \prime}$ & $143^{\circ} 19^{\prime} 12^{\prime \prime}$ & 3.07 & $5: 20$ & -0.35 & Hir & 2.7 \\
\hline Hiroo & $42^{\circ} 17^{\prime} 22^{\prime \prime}$ & $143^{\circ} 19^{\prime} 12^{\prime \prime}$ & 3.10 & $5: 20$ & -0.35 & Hir & 2.8 \\
\hline Hiroo & $42^{\circ} 17^{\prime} 22^{\prime \prime}$ & $143^{\circ} 19^{\prime} 12^{\prime \prime}$ & 3.48 & $5: 20$ & -0.35 & Hir & 3.1 \\
\hline Hiroo & $42^{\circ} 17^{\prime} 19^{\prime \prime}$ & $143^{\circ} 19^{\prime} 06^{\prime \prime}$ & 3.00 & $5: 20$ & -0.40 & Hir & 2.6 \\
\hline Hiroo & $42^{\circ} 17^{\prime} 19^{\prime \prime}$ & $143^{\circ} 19^{\prime} 06^{\prime \prime}$ & 2.99 & $5: 20$ & -0.40 & Hir & 2.6 \\
\hline Hiroo & $42^{\circ} 17^{\prime} 19^{\prime \prime}$ & $143^{\circ} 19^{\prime} 06^{\prime \prime}$ & 2.99 & $5: 20$ & -0.40 & Hir & 2.6 \\
\hline Hiroo & $42^{\circ} 17^{\prime} 19^{\prime \prime}$ & $143^{\circ} 19^{\prime} 06^{\prime \prime}$ & 3.03 & $5: 20$ & -0.40 & Hir & 2.6 \\
\hline Hiroo & $42^{\circ} 17^{\prime} 19^{\prime \prime}$ & $143^{\circ} 19^{\prime} 06^{\prime \prime}$ & 3.00 & $5: 20$ & -0.40 & Hir & 2.6 \\
\hline Hiroo & $42^{\circ} 17^{\prime} 19^{\prime \prime}$ & $143^{\circ} 19^{\prime} 06^{\prime \prime}$ & 3.00 & $5: 20$ & -0.40 & Hir & 2.6 \\
\hline Hiroo & $42^{\circ} 17^{\prime} 19^{\prime \prime}$ & $143^{\circ} 19^{\prime} 06^{\prime \prime}$ & 2.96 & $5: 20$ & -0.40 & Hir & 2.6 \\
\hline Hiroo & $42^{\circ} 17^{\prime} 19^{\prime \prime}$ & $143^{\circ} 19^{\prime} 06^{\prime \prime}$ & 2.95 & $5: 20$ & -0.40 & Hir & 2.6 \\
\hline Hiroo & $42^{\circ} 17^{\prime} 19^{\prime \prime}$ & $143^{\circ} 19^{\prime} 06^{\prime \prime}$ & 2.98 & $5: 20$ & -0.40 & Hir & 2.6 \\
\hline Hiroo & $42^{\circ} 17^{\prime} 19^{\prime \prime}$ & $143^{\circ} 19^{\prime} 06^{\prime \prime}$ & 3.08 & $5: 20$ & -0.40 & Hir & 2.7 \\
\hline Hiroo & $42^{\circ} 17^{\prime} 19^{\prime \prime}$ & $143^{\circ} 19^{\prime} 06^{\prime \prime}$ & 3.08 & $5: 20$ & -0.40 & Hir & 2.7 \\
\hline Hiroo & $42^{\circ} 17^{\prime} 19^{\prime \prime}$ & $143^{\circ} 19^{\prime} 06^{\prime \prime}$ & 2.89 & $5: 20$ & -0.40 & Hir & 2.5 \\
\hline Hiroo & $42^{\circ} 17^{\prime} 19^{\prime \prime}$ & $143^{\circ} 19^{\prime} 06^{\prime \prime}$ & 3.15 & $5: 20$ & -0.45 & Hir & 2.7 \\
\hline Hiroo & $42^{\circ} 17^{\prime} 50^{\prime \prime}$ & $143^{\circ} 19^{\prime} 22^{\prime \prime}$ & 3.14 & $5: 20$ & -0.45 & Hir & 2.7 \\
\hline Hiroo & $42^{\circ} 17^{\prime} 20^{\prime \prime}$ & $143^{\circ} 19^{\prime} 17^{\prime \prime}$ & 2.07 & $5: 20$ & 0.45 & Hir & 2.5 \\
\hline Hiroo & $42^{\circ} 17^{\prime} 20^{\prime \prime}$ & $143^{\circ} 19^{\prime} 17^{\prime \prime}$ & 2.55 & $5: 20$ & 0.45 & Hir & 3.0 \\
\hline Hiroo & $42^{\circ} 17^{\prime} 22^{\prime \prime}$ & $143^{\circ} 19^{\prime} 17^{\prime \prime}$ & 2.05 & $5: 20$ & 0.50 & Hir & 2.6 \\
\hline
\end{tabular}


Table 1. (continued).

\begin{tabular}{|c|c|c|c|c|c|c|c|}
\hline survey point & $\begin{array}{l}\text { latitude } \\
(\mathrm{N})\end{array}$ & $\begin{array}{l}\text { longitude } \\
\text { (E) }\end{array}$ & $\begin{array}{c}\text { measured } \\
\text { height } \\
\text { (m) }\end{array}$ & $\begin{array}{c}\text { tsunami } \\
\text { arrival } \\
\text { time }^{1)}\end{array}$ & $\begin{array}{c}\text { tidal } \\
\text { correction } \\
\text { (m) }\end{array}$ & $\begin{array}{l}\text { reference } \\
\text { tide } \\
\text { gauge }^{2)}\end{array}$ & $\begin{array}{c}\text { corrected } \\
\text { height } \\
\text { (m) }\end{array}$ \\
\hline Shinseikaigan & $42^{\circ} 20^{\prime} 27^{\prime \prime}$ & $143^{\circ} 20^{\prime} 29^{\prime \prime}$ & 2.29 & $5: 20$ & 0.05 & Hir & 2.3 \\
\hline Toyoni-gawa & $42^{\circ} 23^{\prime} 11^{\prime \prime}$ & $143^{\circ} 22^{\prime} 08^{\prime \prime}$ & 3.13 & $5: 20$ & -0.40 & Hir & 2.7 \\
\hline Asahihama & $42^{\circ} 25^{\prime} 09^{\prime \prime}$ & $143^{\circ} 23^{\prime} 30^{\prime \prime}$ & 1.39 & $5: 20$ & 0.30 & Hir & 1.7 \\
\hline Asahihama & $42^{\circ} 26^{\prime} 12^{\prime \prime}$ & $143^{\circ} 24^{\prime} 11^{\prime \prime}$ & 2.94 & $5: 20$ & 0.30 & Hir & 3.2 \\
\hline Asahihama & $42^{\circ} 26^{\prime} 13^{\prime \prime}$ & $143^{\circ} 24^{\prime} 13^{\prime \prime}$ & 3.26 & $5: 20$ & -0.40 & Hir & 2.9 \\
\hline Hamataiki & $42^{\circ} 28^{\prime} 12^{\prime \prime}$ & $143^{\circ} 25^{\prime} 46^{\prime \prime}$ & 3.28 & $5: 20$ & 0.10 & Hir & 3.4 \\
\hline Hamataiki & $42^{\circ} 28^{\prime} 17^{\prime \prime}$ & $143^{\circ} 25^{\prime} 48^{\prime \prime}$ & 3.00 & $5: 20$ & 0.25 & Hir & 3.3 \\
\hline Hamataiki & $42^{\circ} 28^{\prime} 37^{\prime \prime}$ & $143^{\circ} 25^{\prime} 45^{\prime \prime}$ & 2.38 & $5: 20$ & -0.05 & Hir & 2.3 \\
\hline Hamataiki & $42^{\circ} 28^{\prime} 35^{\prime \prime}$ & $143^{\circ} 25^{\prime} 53^{\prime \prime}$ & 2.62 & $5: 20$ & 0.45 & Hir & 3.1 \\
\hline Hamataiki & $42^{\circ} 28^{\prime} 30^{\prime \prime}$ & $143^{\circ} 25^{\prime} 56^{\prime \prime}$ & 3.00 & $5: 20$ & -0.40 & Hir & 2.6 \\
\hline Hamataiki & $42^{\circ} 28^{\prime} 37^{\prime \prime}$ & $143^{\circ} 26^{\prime} 00^{\prime \prime}$ & 3.92 & $5: 20$ & 0.05 & Hir & 4.0 \\
\hline Horokayantou & $42^{\circ} 31^{\prime} 29^{\prime \prime}$ & $143^{\circ} 28^{\prime} 40^{\prime \prime}$ & 4.42 & $5: 20$ & -0.40 & Hir & 4.0 \\
\hline Horokayantou & $42^{\circ} 31^{\prime} 30^{\prime \prime}$ & $143^{\circ} 28^{\prime} 41^{\prime \prime}$ & 3.69 & $5: 20$ & -0.30 & Hir & 3.4 \\
\hline Banseionsen & $42^{\circ} 31^{\prime} 58^{\prime \prime}$ & $143^{\circ} 29^{\prime} 09^{\prime \prime}$ & 4.49 & $5: 20$ & -0.40 & Hir & 4.1 \\
\hline Banseionsen & $42^{\circ} 32^{\prime} 01^{\prime \prime}$ & $143^{\circ} 29^{\prime} 11^{\prime \prime}$ & 3.20 & $5: 20$ & 0.10 & Hir & 3.3 \\
\hline Banseionsen & $42^{\circ} 32^{\prime} 09^{\prime \prime}$ & $143^{\circ} 29^{\prime} 20^{\prime \prime}$ & 2.63 & $5: 20$ & 0.40 & Hir & 3.0 \\
\hline Oikamanai & $42^{\circ} 33^{\prime} 28^{\prime \prime}$ & $143^{\circ} 30^{\prime} 31^{\prime \prime}$ & 4.28 & $5: 20$ & -0.25 & Hir & 4.0 \\
\hline Oikamanai & $42^{\circ} 33^{\prime} 34^{\prime \prime}$ & $143^{\circ} 30^{\prime} 39^{\prime \prime}$ & 2.40 & $5: 20$ & 0.45 & Hir & 2.9 \\
\hline Yudonuma & $42^{\circ} 34^{\prime} 49^{\prime \prime}$ & $143^{\circ} 31^{\prime} 50^{\prime \prime}$ & 2.58 & $5: 20$ & 0.05 & Hir & 2.6 \\
\hline Yudonuma & $42^{\circ} 35^{\prime} 07^{\prime \prime}$ & $143^{\circ} 32^{\prime} 09^{\prime \prime}$ & 3.07 & $5: 20$ & -0.45 & Hir & 2.6 \\
\hline Yudonuma & $42^{\circ} 35^{\prime} 57^{\prime \prime}$ & $143^{\circ} 33^{\prime} 00^{\prime \prime}$ & 3.35 & $5: 20$ & -0.05 & Hir & 3.3 \\
\hline Yudonuma & $42^{\circ} 36^{\prime} 00^{\prime \prime}$ & $143^{\circ} 33^{\prime} 05^{\prime \prime}$ & 3.30 & $5: 20$ & -0.05 & Hir & 3.3 \\
\hline Yudonuma & $42^{\circ} 36^{\prime} 39^{\prime \prime}$ & $143^{\circ} 33^{\prime} 46^{\prime \prime}$ & 2.40 & $5: 20$ & 0.60 & Hir & 3.0 \\
\hline Cyosetsunuma & $42^{\circ} 39^{\prime} 17^{\prime \prime}$ & $143^{\circ} 36^{\prime} 45^{\prime \prime}$ & 3.16 & $5: 20$ & 0.30 & Hir & 3.5 \\
\hline Cyosetsunuma & $42^{\circ} 39^{\prime} 37^{\prime \prime}$ & $143^{\circ} 37^{\prime} 11^{\prime \prime}$ & 3.52 & $5: 20$ & -0.05 & Hir & 3.5 \\
\hline Ootsu-gyoko & $42^{\circ} 40^{\prime} 14^{\prime \prime}$ & $143^{\circ} 37^{\prime} 59^{\prime \prime}$ & 2.62 & $5: 20$ & 0.15 & Hir, Kus & 2.8 \\
\hline Ootsu-gyoko & $42^{\circ} 40^{\prime} 50^{\prime \prime}$ & $143^{\circ} 38^{\prime} 05^{\prime \prime}$ & 2.24 & $5: 20$ & 0.45 & Hir, Kus & 2.7 \\
\hline Ootsu-gyoko & $42^{\circ} 40^{\prime} 50^{\prime \prime}$ & $143^{\circ} 38^{\prime} 05^{\prime \prime}$ & 2.30 & $5: 20$ & 0.45 & Hir, Kus & 2.8 \\
\hline Ootsu-gyoko & $42^{\circ} 40^{\prime} 50^{\prime \prime}$ & $143^{\circ} 38^{\prime} 05^{\prime \prime}$ & 2.21 & $5: 20$ & 0.45 & Hir, Kus & 2.7 \\
\hline Ootsu-gyoko & $42^{\circ} 40^{\prime} 50^{\prime \prime}$ & $143^{\circ} 38^{\prime} 05^{\prime \prime}$ & 2.20 & $5: 20$ & 0.45 & Hir, Kus & 2.7 \\
\hline Ootsu-gyoko & $42^{\circ} 40^{\prime} 50^{\prime \prime}$ & $143^{\circ} 38^{\prime} 05^{\prime \prime}$ & 2.32 & $5: 20$ & 0.45 & Hir, Kus & 2.8 \\
\hline Ootsu-gyoko & $42^{\circ} 40^{\prime} 50^{\prime \prime}$ & $143^{\circ} 38^{\prime} 05^{\prime \prime}$ & 2.30 & $5: 20$ & 0.45 & Hir, Kus & 2.8 \\
\hline Ootsu-gyoko & $42^{\circ} 40^{\prime} 50^{\prime \prime}$ & $143^{\circ} 38^{\prime} 05^{\prime \prime}$ & 2.32 & $5: 20$ & 0.45 & Hir, Kus & 2.8 \\
\hline Ootsu-gyoko & $42^{\circ} 40^{\prime} 50^{\prime \prime}$ & $143^{\circ} 38^{\prime} 05^{\prime \prime}$ & 2.39 & $5: 20$ & 0.45 & Hir, Kus & 2.8 \\
\hline Ootsu-gyoko & $42^{\circ} 40^{\prime} 29^{\prime \prime}$ & $143^{\circ} 38^{\prime} 31^{\prime \prime}$ & 1.97 & $5: 20$ & 0.35 & Hir, Kus & 2.3 \\
\hline Ootsu-gyoko & $42^{\circ} 40^{\prime} 41^{\prime \prime}$ & $143^{\circ} 38^{\prime} 44^{\prime \prime}$ & 2.43 & $5: 20$ & 0.40 & Hir, Kus & 2.8 \\
\hline Ootsu-gyoko & $42^{\circ} 40^{\prime} 50^{\prime \prime}$ & $143^{\circ} 38^{\prime} 05^{\prime \prime}$ & 2.53 & $5: 20$ & 0.00 & Hir, Kus & 2.5 \\
\hline Ootsu-gyoko & $42^{\circ} 40^{\prime} 50^{\prime \prime}$ & $143^{\circ} 38^{\prime} 05^{\prime \prime}$ & 2.50 & $5: 20$ & 0.00 & Hir, Kus & 2.5 \\
\hline Ootsu-gyoko & $42^{\circ} 40^{\prime} 50^{\prime \prime}$ & $143^{\circ} 38^{\prime} 05^{\prime \prime}$ & 3.24 & $5: 20$ & 0.05 & Hir, Kus & 3.3 \\
\hline Ootsu-gyoko & $42^{\circ} 40^{\prime} 50^{\prime \prime}$ & $143^{\circ} 38^{\prime} 05^{\prime \prime}$ & 2.09 & $5: 20$ & 0.15 & Hir, Kus & 2.2 \\
\hline Ootsu-gyoko & $42^{\circ} 40^{\prime} 50^{\prime \prime}$ & $143^{\circ} 38^{\prime} 05^{\prime \prime}$ & 2.05 & $5: 20$ & 0.15 & Hir, Kus & 2.2 \\
\hline Ootsu-gyoko & $42^{\circ} 40^{\prime} 50^{\prime \prime}$ & $143^{\circ} 38^{\prime} 29^{\prime \prime}$ & 2.78 & $5: 20$ & -0.05 & Hir, Kus & 2.7 \\
\hline Ootsu-gyoko & $42^{\circ} 40^{\prime} 50^{\prime \prime}$ & $143^{\circ} 38^{\prime} 29^{\prime \prime}$ & 2.58 & $5: 20$ & 0.05 & Hir, Kus & 2.6 \\
\hline Ootsu-gyoko & $42^{\circ} 40^{\prime} 42^{\prime \prime}$ & $143^{\circ} 38^{\prime} 02^{\prime \prime}$ & 2.60 & $5: 20$ & 0.00 & Hir, Kus & 2.6 \\
\hline Ootsu-gyoko & $42^{\circ} 40^{\prime} 42^{\prime \prime}$ & $143^{\circ} 38^{\prime} 02^{\prime \prime}$ & 2.32 & $5: 20$ & 0.00 & Hir, Kus & 2.3 \\
\hline Ootsu-gyoko & $42^{\circ} 40^{\prime} 42^{\prime \prime}$ & $143^{\circ} 38^{\prime} 02^{\prime \prime}$ & 2.47 & $5: 20$ & 0.00 & Hir, Kus & 2.5 \\
\hline Ootsu-gyoko & $42^{\circ} 40^{\prime} 42^{\prime \prime}$ & $143^{\circ} 38^{\prime} 21^{\prime \prime}$ & 1.65 & $5: 20$ & 0.00 & Hir, Kus & 1.7 \\
\hline Ootsu-gyoko & $42^{\circ} 41^{\prime} 00^{\prime \prime}$ & $143^{\circ} 38^{\prime} 47^{\prime \prime}$ & 2.29 & $5: 20$ & 0.00 & Hir, Kus & 2.3 \\
\hline Ootsu-gyoko & $42^{\circ} 41^{\prime} 00^{\prime \prime}$ & $143^{\circ} 38^{\prime} 47^{\prime \prime}$ & 2.54 & $5: 20$ & 0.00 & Hir, Kus & 2.5 \\
\hline Ootsu-gyoko & $42^{\circ} 41^{\prime} 00^{\prime \prime}$ & $143^{\circ} 38^{\prime} 47^{\prime \prime}$ & 2.21 & $5: 20$ & 0.25 & Hir, Kus & 2.5 \\
\hline Ootsu-gyoko & $42^{\circ} 41^{\prime} 00^{\prime \prime}$ & $143^{\circ} 38^{\prime} 47^{\prime \prime}$ & 2.51 & $5: 20$ & 0.25 & Hir, Kus & 2.8 \\
\hline Ootsu-gyoko & $42^{\circ} 40^{\prime} 35^{\prime \prime}$ & $143^{\circ} 38^{\prime} 13^{\prime \prime}$ & 2.75 & $5: 20$ & -0.50 & Hir, Kus & 2.3 \\
\hline Ootsu-gyoko & $42^{\circ} 40^{\prime} 50^{\prime \prime}$ & $143^{\circ} 38^{\prime} 29^{\prime \prime}$ & 2.90 & $5: 20$ & -0.40 & Hir, Kus & 2.5 \\
\hline Ootsu-gyoko & $42^{\circ} 40^{\prime} 50^{\prime \prime}$ & $143^{\circ} 38^{\prime} 05^{\prime \prime}$ & 3.50 & $5: 20$ & -0.50 & Hir, Kus & 3.0 \\
\hline Ootsu-gyoko & $42^{\circ} 40^{\prime} 50^{\prime \prime}$ & $143^{\circ} 38^{\prime} 05^{\prime \prime}$ & 3.40 & $5: 20$ & -0.50 & Hir, Kus & 2.9 \\
\hline Ootsu-gyoko & $42^{\circ} 40^{\prime} 50^{\prime \prime}$ & $143^{\circ} 38^{\prime} 05^{\prime \prime}$ & 3.65 & $5: 20$ & -0.50 & Hir, Kus & 3.2 \\
\hline Ootsu-gyoko & $42^{\circ} 40^{\prime} 50^{\prime \prime}$ & $143^{\circ} 38^{\prime} 05^{\prime \prime}$ & 3.25 & $5: 20$ & -0.50 & Hir, Kus & 2.8 \\
\hline Ootsu-gyoko & $42^{\circ} 40^{\prime} 50^{\prime \prime}$ & $143^{\circ} 38^{\prime} 05^{\prime \prime}$ & 2.95 & $5: 20$ & -0.50 & Hir, Kus & 2.5 \\
\hline Ootsu-gyoko & $42^{\circ} 40^{\prime} 50^{\prime \prime}$ & $143^{\circ} 8^{\prime} 05^{\prime \prime}$ & 3.10 & $5: 20$ & -0.30 & Hir, Kus & 2.8 \\
\hline Ootsu-gyoko & $42^{\circ} 40^{\prime} 50^{\prime \prime}$ & $143^{\circ} 38^{\prime} 05^{\prime \prime}$ & 3.00 & $5: 20$ & -0.30 & Hir, Kus & 2.7 \\
\hline Ootsu-gyoko & $42^{\circ} 40^{\prime} 50^{\prime \prime}$ & $143^{\circ} 38^{\prime} 05^{\prime \prime}$ & 2.80 & $5: 20$ & -0.25 & Hir, Kus & 2.6 \\
\hline Ootsu-gyoko & $42^{\circ} 40^{\prime} 50^{\prime \prime}$ & $143^{\circ} 38^{\prime} 05^{\prime \prime}$ & 2.70 & $5: 20$ & -0.20 & Hir, Kus & 2.5 \\
\hline Ootsu-gyoko & $42^{\circ} 40^{\prime} 40^{\prime \prime}$ & $143^{\circ} 38^{\prime} 18^{\prime \prime}$ & 2.95 & $5: 20$ & -0.30 & Hir, Kus & 2.7 \\
\hline Ootsu-gyoko & $42^{\circ} 40^{\prime} 39^{\prime \prime}$ & $143^{\circ} 38^{\prime} 05^{\prime \prime}$ & 2.31 & $5: 20$ & -0.05 & Hir, Kus & 2.3 \\
\hline Ootsu-gyoko & $42^{\circ} 40^{\prime} 39^{\prime \prime}$ & $143^{\circ} 38^{\prime} 05^{\prime \prime}$ & 1.77 & $5: 20$ & -0.05 & Hir, Kus & 1.7 \\
\hline Ootsu-gyoko & $42^{\circ} 40^{\prime} 38^{\prime \prime}$ & $143^{\circ} 38^{\prime} 35^{\prime \prime}$ & 2.27 & $5: 20$ & -0.40 & Hir, Kus & 1.9 \\
\hline Ootsu-gyoko & $42^{\circ} 40^{\prime} 41^{\prime \prime}$ & $143^{\circ} 38^{\prime} 37^{\prime \prime}$ & 2.55 & $5: 20$ & -0.40 & Hir, Kus & 2.2 \\
\hline Tokachi-gawa & $42^{\circ} 41^{\prime} 26^{\prime \prime}$ & $143^{\circ} 39^{\prime} 34^{\prime \prime}$ & 2.03 & $5: 20$ & -0.25 & Hir, Kus & 1.8 \\
\hline Tokachibuto & $42^{\circ} 42^{\prime} 30^{\prime \prime}$ & $143^{\circ} 40^{\prime} 57^{\prime \prime}$ & 2.83 & $5: 20$ & -0.15 & Hir, Kus & 2.7 \\
\hline Hamaatsunai & $42^{\circ} 47^{\prime} 29^{\prime \prime}$ & $143^{\circ} 48^{\prime} 05^{\prime \prime}$ & 2.93 & $5: 20$ & -0.40 & Hir, Kus & 2.5 \\
\hline Atsunai-gyoko & $42^{\circ} 48^{\prime} 25^{\prime \prime}$ & $143^{\circ} 49^{\prime} 11^{\prime \prime}$ & 1.02 & $5: 20$ & 0.20 & Hir, Kus & 1.2 \\
\hline Atsunai-gyoko & $42^{\circ} 48^{\prime} 25^{\prime \prime}$ & $143^{\circ} 49^{\prime} 11^{\prime \prime}$ & 2.27 & $5: 20$ & -0.40 & Hir, Kus & 1.9 \\
\hline Atsunai-gyoko & $42^{\circ} 48^{\prime} 25^{\prime \prime}$ & $143^{\circ} 49^{\prime} 11^{\prime \prime}$ & 1.82 & $5: 20$ & 0.55 & Hir, Kus & 2.4 \\
\hline Atsunai-gyoko & $42^{\circ} 48^{\prime} 27^{\prime \prime}$ & $143^{\circ} 49^{\prime} 16^{\prime \prime}$ & 0.91 & $5: 20$ & 0.20 & Hir, Kus & 1.1 \\
\hline Atsunai-gyoko & $42^{\circ} 48^{\prime} 30^{\prime \prime}$ & $143^{\circ} 49^{\prime} 21^{\prime \prime}$ & 2.03 & $5: 20$ & 0.10 & Hir, Kus & 2.1 \\
\hline Kinashibetsu & $42^{\circ} 51^{\prime} 12^{\prime \prime}$ & $143^{\circ} 53^{\prime} 03^{\prime \prime}$ & 2.25 & $5: 20$ & -0.10 & Hir, Kus & 2.2 \\
\hline Otobetsuhigashi & $42^{\circ} 55^{\prime} 16^{\prime \prime}$ & $143^{\circ} 59^{\prime} 49^{\prime \prime}$ & 1.11 & $5: 20$ & 0.40 & Hir, Kus & 1.5 \\
\hline
\end{tabular}


Table 1. (continued).

\begin{tabular}{|c|c|c|c|c|c|c|c|}
\hline survey point & $\begin{array}{l}\text { latitude } \\
(\mathrm{N})\end{array}$ & $\begin{array}{l}\text { longitude } \\
\text { (E) }\end{array}$ & $\begin{array}{c}\text { measured } \\
\text { height } \\
\text { (m) }\end{array}$ & $\begin{array}{c}\text { tsunami } \\
\text { arrival } \\
\text { time }\end{array}$ & $\begin{array}{l}\text { tidal } \\
\text { correction } \\
(\mathrm{m})\end{array}$ & $\begin{array}{c}\text { reference } \\
\text { tide } \\
\text { gauge }^{2)}\end{array}$ & $\begin{array}{c}\text { corrected } \\
\text { height } \\
\text { (m) }\end{array}$ \\
\hline Pasyukurunuma & $42^{\circ} 55^{\prime} 57^{\prime \prime}$ & $144^{\circ} 00^{\prime} 04^{\prime \prime}$ & 2.42 & $5: 20$ & -0.10 & Hir, Kus & 2.3 \\
\hline Sarogawa & $42^{\circ} 57^{\prime} 19^{\prime \prime}$ & $144^{\circ} 03^{\prime} 43^{\prime \prime}$ & 1.54 & $5: 20$ & 0.40 & Hir, Kus & 1.9 \\
\hline Sarogawa & $42^{\circ} 57^{\prime} 19^{\prime \prime}$ & $144^{\circ} 03^{\prime} 43^{\prime \prime}$ & 2.27 & $5: 20$ & 0.35 & Hir, Kus & 2.6 \\
\hline Sarogawa & $42^{\circ} 57^{\prime} 03^{\prime \prime}$ & $144^{\circ} 03^{\prime} 43^{\prime \prime}$ & 1.39 & $5: 20$ & 0.45 & Hir, Kus & 1.8 \\
\hline Shiranuka-gyoko & $42^{\circ} 57^{\prime} 11^{\prime \prime}$ & $144^{\circ} 05^{\prime} 12^{\prime \prime}$ & 1.50 & $5: 20$ & 0.15 & Kus & 1.7 \\
\hline Shiranuka-gyoko & $42^{\circ} 57^{\prime} 12^{\prime \prime}$ & $144^{\circ} 05^{\prime} 12^{\prime \prime}$ & 2.11 & $5: 20$ & 0.40 & Kus & 2.5 \\
\hline Koitoi & $42^{\circ} 59^{\prime} 41^{\prime \prime}$ & $144^{\circ} 11^{\prime} 45^{\prime \prime}$ & 1.62 & $5: 20$ & 0.35 & Kus & 2.0 \\
\hline Kushiro-ko & $43^{\circ} 00^{\prime} 01^{\prime \prime}$ & $144^{\circ} 19^{\prime} 48^{\prime \prime}$ & 1.20 & $5: 20$ & 0.40 & Kus & 1.6 \\
\hline Kushiro-ko & $43^{\circ} 00^{\prime} 01^{\prime \prime}$ & $144^{\circ} 19^{\prime} 48^{\prime \prime}$ & 1.25 & $5: 20$ & 0.35 & Kus & 1.6 \\
\hline Kushiro-ko & $42^{\circ} 59^{\prime} 00^{\prime \prime}$ & $144^{\circ} 22^{\prime} 16^{\prime \prime}$ & 1.46 & $5: 20$ & -0.10 & Kus & 1.4 \\
\hline Kushiro-ko & $42^{\circ} 58^{\prime} 54^{\prime \prime}$ & $144^{\circ} 22^{\prime} 41^{\prime \prime}$ & 1.38 & $5: 20$ & -0.10 & Kus & 1.3 \\
\hline Kushiro-ko & $42^{\circ} 58^{\prime} 54^{\prime \prime}$ & $144^{\circ} 22^{\prime} 41^{\prime \prime}$ & 0.85 & $5: 20$ & 0.40 & Kus & 1.3 \\
\hline Kushiro-ko & $42^{\circ} 58^{\prime} 54^{\prime \prime}$ & $144^{\circ} 22^{\prime} 41^{\prime \prime}$ & 0.90 & $5: 20$ & 0.40 & Kus & 1.3 \\
\hline Kushiro-ko & $42^{\circ} 58^{\prime} 54^{\prime \prime}$ & $144^{\circ} 22^{\prime} 41^{\prime \prime}$ & 1.00 & $5: 20$ & 0.40 & Kus & 1.4 \\
\hline Chiyonoura & $42^{\circ} 58^{\prime} 00^{\prime \prime}$ & $144^{\circ} 23^{\prime} 35^{\prime \prime}$ & 1.16 & $5: 20$ & 0.00 & Kus & 1.2 \\
\hline Kushiro-gawa & $43^{\circ} 00^{\prime} 24^{\prime \prime}$ & $144^{\circ} 23^{\prime} 55^{\prime \prime}$ & 1.35 & $5: 20$ & 0.10 & Kus & 1.5 \\
\hline Konbumori & $42^{\circ} 57^{\prime} 03^{\prime \prime}$ & $144^{\circ} 31^{\prime} 49^{\prime \prime}$ & 1.22 & $6: 00$ & 0.10 & Kus & 1.3 \\
\hline Konbumori & $42^{\circ} 57^{\prime} 53^{\prime \prime}$ & $144^{\circ} 31^{\prime} 51^{\prime \prime}$ & 0.42 & $6: 00$ & 0.65 & Kus & 1.1 \\
\hline Fushiko & $42^{\circ} 56^{\prime} 58^{\prime \prime}$ & $144^{\circ} 32^{\prime} 25^{\prime \prime}$ & 1.50 & $6: 00$ & 0.15 & Kus & 1.7 \\
\hline Pondomari & $42^{\circ} 57^{\prime} 04^{\prime \prime}$ & $144^{\circ} 37^{\prime} 02^{\prime \prime}$ & 1.55 & $6: 00$ & 0.05 & Kus & 1.6 \\
\hline Chippomanai & $42^{\circ} 56^{\prime} 01^{\prime \prime}$ & $144^{\circ} 44^{\prime} 42^{\prime \prime}$ & 1.03 & $6: 30$ & 0.65 & Kus, Akk & 1.7 \\
\hline Senposhi & $42^{\circ} 58^{\prime} 33^{\prime \prime}$ & $144^{\circ} 43^{\prime} 21^{\prime \prime}$ & 0.86 & $6: 30$ & 0.85 & Akk & 1.7 \\
\hline Monshizu & $43^{\circ} 03^{\prime} 15^{\prime \prime}$ & $144^{\circ} 46^{\prime} 54^{\prime \prime}$ & 1.18 & $6: 30$ & 0.85 & Akk & 2.0 \\
\hline Akkeshi-ko & $43^{\circ} 03^{\prime} 00^{\prime \prime}$ & $144^{\circ} 51^{\prime} 01^{\prime \prime}$ & 0.46 & $6: 30$ & 0.85 & Akk & 1.3 \\
\hline Tsukushikoi & $43^{\circ} 00^{\prime} 54^{\prime \prime}$ & $144^{\circ} 50^{\prime} 47^{\prime \prime}$ & 2.28 & $6: 30$ & -0.30 & Akk & 2.0 \\
\hline Tokotan & $42^{\circ} 59^{\prime} 35^{\prime \prime}$ & $144^{\circ} 52^{\prime} 27^{\prime \prime}$ & 2.35 & $6: 30$ & 0.75 & Akk & 3.1 \\
\hline Tokotan & $42^{\circ} 59^{\prime} 44^{\prime \prime}$ & $144^{\circ} 52^{\prime} 07^{\prime \prime}$ & 2.20 & $6: 30$ & 0.50 & Akk & 2.7 \\
\hline Tokotan & $42^{\circ} 59^{\prime} 35^{\prime \prime}$ & $144^{\circ} 52^{\prime} 18^{\prime \prime}$ & 1.84 & $6: 30$ & 0.10 & Akk & $\begin{array}{l}2.1 \\
1.9\end{array}$ \\
\hline Horomanbetsu & $42^{\circ} 59^{\prime} 14^{\prime \prime}$ & $144^{\circ} 53^{\prime} 16^{\prime \prime}$ & 1.70 & $6: 30$ & 0.55 & Akk & 2.3 \\
\hline Mabiro & $42^{\circ} 59^{\prime} 20^{\prime \prime}$ & $144^{\circ} 53^{\prime} 50^{\prime \prime}$ & 3.74 & $6: 30$ & 0.25 & Akk & 4.0 \\
\hline Mabiro & $42^{\circ} 59^{\prime} 20^{\prime \prime}$ & $144^{\circ} 54^{\prime} 24^{\prime \prime}$ & 2.88 & $6: 30$ & 0.45 & Akk & 3.3 \\
\hline Mabiro & $42^{\circ} 59^{\prime} 20^{\prime \prime}$ & $144^{\circ} 54^{\prime} 04^{\prime \prime}$ & 3.92 & $6: 30$ & 0.50 & Akk & 4.4 \\
\hline Mabiro & $42^{\circ} 59^{\prime} 22^{\prime \prime}$ & $144^{\circ} 53^{\prime} 53^{\prime \prime}$ & 3.76 & $6: 30$ & 0.50 & Akk & 4.3 \\
\hline Mabiro & $42^{\circ} 59^{\prime} 19^{\prime \prime}$ & $144^{\circ} 54^{\prime} 19^{\prime \prime}$ & 2.28 & $6: 30$ & 0.00 & Akk & 2.3 \\
\hline Mabiro & $42^{\circ} 59^{\prime} 17^{\prime \prime}$ & $144^{\circ} 54^{\prime} 23^{\prime \prime}$ & 1.94 & $6: 30$ & 0.00 & Akk & 1.9 \\
\hline Mabiro & $42^{\circ} 59^{\prime} 20^{\prime \prime}$ & $144^{\circ} 54^{\prime} 17^{\prime \prime}$ & 2.33 & $6: 30$ & 0.00 & Akk & 2.3 \\
\hline Mochirippu & $43^{\circ} 01^{\prime} 17^{\prime \prime}$ & $145^{\circ} 01^{\prime} 16^{\prime \prime}$ & 1.00 & $6: 30$ & 0.20 & Akk, Och & 1.2 \\
\hline Hichirippu & $43^{\circ} 01^{\prime} 49^{\prime \prime}$ & $145^{\circ} 01^{\prime} 39^{\prime \prime}$ & 1.20 & $6: 30$ & 0.05 & Akk, Och & 1.3 \\
\hline Biwase & $43^{\circ} 03^{\prime} 33^{\prime \prime}$ & $145^{\circ} 05^{\prime} 16^{\prime \prime}$ & 1.49 & $6: 30$ & 0.00 & Akk, Och & 1.5 \\
\hline Shinkawa & $43^{\circ} 04^{\prime} 48^{\prime \prime}$ & $145^{\circ} 06^{\prime} 36^{\prime \prime}$ & 1.80 & $6: 30$ & 0.00 & Akk, Och & 1.8 \\
\hline Shinkawa & $43^{\circ} 04^{\prime} 48^{\prime \prime}$ & $145^{\circ} 06^{\prime} 19^{\prime \prime}$ & 0.59 & $6: 30$ & 0.30 & Akk, Och & 0.9 \\
\hline Shinkawa & $43^{\circ} 04^{\prime} 48^{\prime \prime}$ & $145^{\circ} 06^{\prime} 19^{\prime \prime}$ & 0.38 & $6: 30$ & 0.30 & Akk, Och & 0.7 \\
\hline Sakaki-cyou & $43^{\circ} 07^{\prime} 21^{\prime \prime}$ & $145^{\circ} 06^{\prime} 55^{\prime \prime}$ & 1.14 & $6: 30$ & 0.05 & Akk, Och & 1.2 \\
\hline Sakaki-cyou & $43^{\circ} 07^{\prime} 27^{\prime \prime}$ & $145^{\circ} 06^{\prime} 59^{\prime \prime}$ & 1.82 & $6: 30$ & 0.05 & Akk, Och & 1.9 \\
\hline Tofutsu & $43^{\circ} 04^{\prime} 29^{\prime \prime}$ & $145^{\circ} 09^{\prime} 02^{\prime \prime}$ & 1.20 & $6: 30$ & 0.10 & Och & 1.3 \\
\hline Ochiishi & $43^{\circ} 10^{\prime} 40^{\prime \prime}$ & $145^{\circ} 30^{\prime} 29^{\prime \prime}$ & 0.90 & $5: 40$ & 0.45 & Och & 1.4 \\
\hline Cyobushi & $43^{\circ} 15^{\prime} 18^{\prime \prime}$ & $145^{\circ} 33^{\prime} 27^{\prime \prime}$ & 1.82 & $5: 40$ & -0.40 & Han & 1.4 \\
\hline Hanasaki & $43^{\circ} 17^{\prime} 08^{\prime \prime}$ & $145^{\circ} 35^{\prime} 05^{\prime \prime}$ & 1.41 & $5: 40$ & -0.20 & Han & 1.2 \\
\hline Shichikari-gyoko & $41^{\circ} 21^{\prime} 35^{\prime \prime}$ & $141^{\circ} 26^{\prime} 38^{\prime \prime}$ & 2.59 & $5: 50$ & -0.15 & Shi & 2.4 \\
\hline Furuno-gyoko & $41^{\circ} 20^{\prime} 57^{\prime \prime}$ & $141^{\circ} 26^{\prime} 23^{\prime \prime}$ & 1.45 & $5: 50$ & 0.00 & Shi & $\begin{array}{l}2.4 \\
1.5\end{array}$ \\
\hline Tomariminani & $41^{\circ} 03^{\prime} 02^{\prime \prime}$ & $141^{\circ} 23^{\prime} 20^{\prime \prime}$ & 1.50 & $5: 50$ & -0.25 & Shi, Hac & 1.3 \\
\hline Taroo-gyoko & $39^{\circ} 43^{\prime} 45^{\prime \prime}$ & $141^{\circ} 58^{\prime} 25^{\prime \prime}$ & 0.49 & $5: 45$ & 0.05 & Miy & 0.5 \\
\hline Miyako-gyoko & $39^{\circ} 38^{\prime} 23^{\prime \prime}$ & $141^{\circ} 58^{\prime} 04^{\prime \prime}$ & 1.11 & $5: 45$ & -0.25 & Miy & 0.9 \\
\hline Miyako-gyoko & $39^{\circ} 38^{\prime} 29^{\prime \prime}$ & $141^{\circ} 58^{\prime} 04^{\prime \prime}$ & 0.80 & $5: 45$ & -0.10 & Miy & 0.7 \\
\hline Akamae & $39^{\circ} 35^{\prime} 22^{\prime \prime}$ & $141^{\circ} 57^{\prime} 40^{\prime \prime}$ & 0.70 & $5: 45$ & -0.30 & Miy & 0.4 \\
\hline Hirotawan & $39^{\circ} 59^{\prime} 34^{\prime \prime}$ & $141^{\circ} 37^{\prime} 23^{\prime \prime}$ & 1.12 & $5: 45$ & -0.15 & Oof, Kes & 1.0 \\
\hline Kesennuma & $39^{\circ} 53^{\prime} 00^{\prime \prime}$ & $1413^{\circ} 7^{\prime} 30^{\prime \prime}$ & 0.70 & $5: 45$ & -0.15 & Kes & 0.6 \\
\hline
\end{tabular}

1) Tsunami arrival time (in Japan Standard Time of September 26, 2003).

${ }^{2)}$ Reference tide gauges are Hanasaki (Han), Ochiishi (Och), Akkeshi (Akk), Kushiro (Kus), Hiroo (Hir), Utaro (Uta), Urakawa (Ura), Shimokita (Shi), Hachinohe (Hac), Miyako (Miy), Oofunato (Oof), and Kesennuma (Kes).

nally reference to the sea level at the time of measurement. Because those tsunami heights are measured from the sea level at the shore, the error of the measurements is about $0.2 \mathrm{~m}$. The tide levels are corrected using nearby tide gauge records (Hanasaki, Kushiro, Urakawa, Hachinohe, Miyako, and Kesennuma) or the calculated tide data from the tide table provided by the JMA (Ochiishi, Akkeshi, Hiroo, Utaro, Shimokita, and Ofunato). In Table 1, the reference station name is shown for each survey point. If two names are listed for one survey point in Table 1, the average of the two tide data is used. The observed tsunami heights due to the 2003 earthquake in Fig. 1 are calculated from the tide level at the time of the maximum tsunami. The arrival time of the maximum tsunami for each survey point is also listed in Table 1 . Most of the arrival times are obtained from nearby tide gauge records except the survey points between Cape Erimo and Urakawa. The reason that we need to treat those survey points differently is discussed latter. The large tsunamis arrived at the Pacific side of the coast of Hokkaido and Tohoku about 2-3 hours after the time of the high tide. The large 


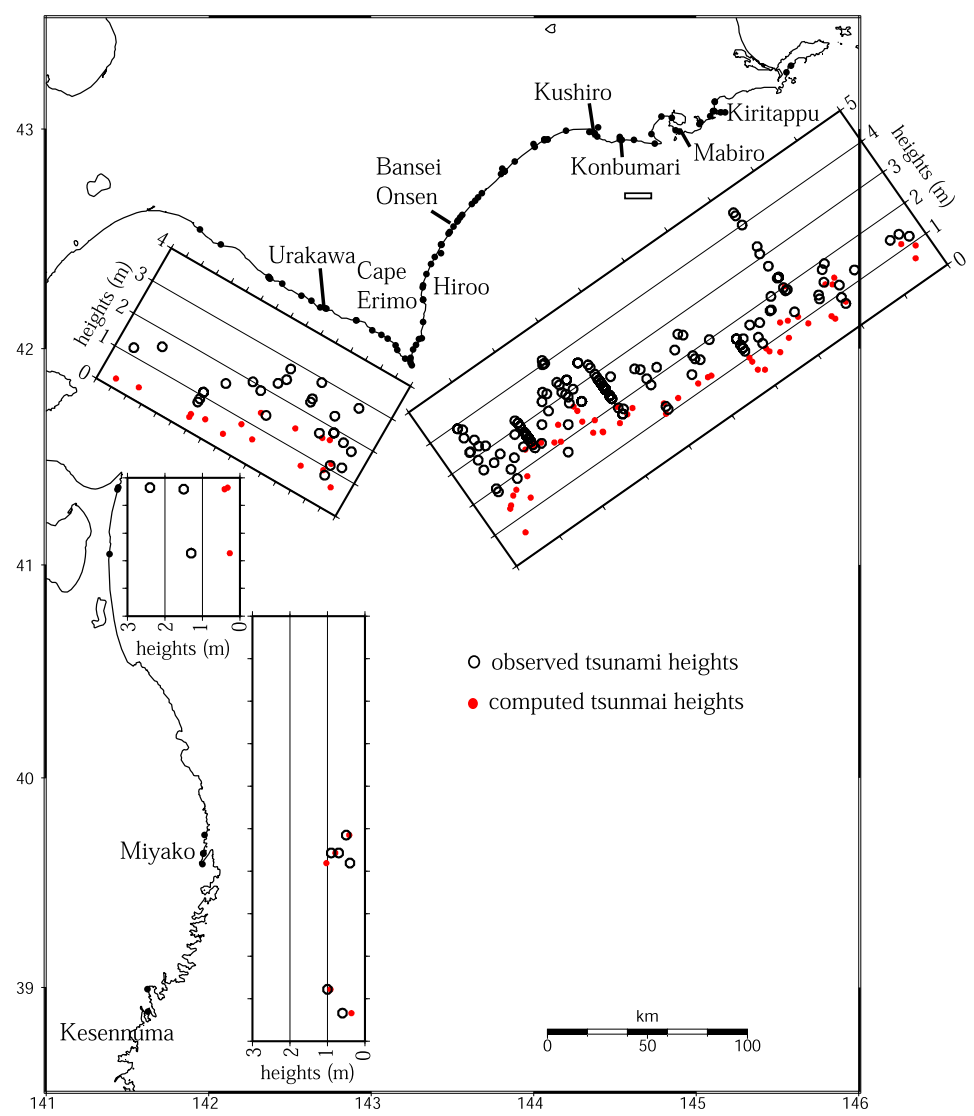

Fig. 1. Comparison of the measured (black circles) and computed (red circles) tsunami heights for the 2003 Tokachi-oki tsunami. The computed tsunami heights are obtained using the slip distribution estimated from the teleseismic body waves (Yamanaka and Kikuchi, 2003).

tsunami run-up heights about $4 \mathrm{~m}$ was observed at the east side of Cape Erimo and Bansei-onsen located about $70 \mathrm{~km}$ north-east of the Cape Erimo. Those places were close to the source region of the earthquake (Fig. 3). In general, the tsunami run-up heights were gradually decreased to the east and to the west away from those two places except Mabiro where the large tsunami height about $4 \mathrm{~m}$ is locally observed.

\section{Numerical Simulation of the Tsunami}

Slip distribution of the 2003 Tokachi-oki earthquake was estimated using the teleseismic body waves by Yamanaka and Kikuchi (2003). The vertical seafloor displacement is computed using Okada's (1985) equations (Fig. 3) and used as an initial condition of tsunami numerical computation. The finite-difference computations of the linear long-wave equations (see Satake, 1995) are carried out. The total reflection boundary condition is used at the coast. The grid size is $20 \mathrm{sec}$ of arc (about $600 \mathrm{~m}$ ). The time step of the computation is $1 \mathrm{sec}$ to satisfy a stability condition. In Fig. 1, the results of the tsunami computation are shown with the observed tsunami heights. The computed tsunami heights slightly underestimate the observed tsunami heights because run-up computations with a finer grid system are not included in this simulation. The overall pattern of the computed tsunami height distribution is similar to the observed one. However, some details between the observed and computed tsunami heights are different. One is that the computed tsunamis largely underestimate the observed tsunami heights, about $4 \mathrm{~m}$, to the east of Cape Erimo. Another is a)

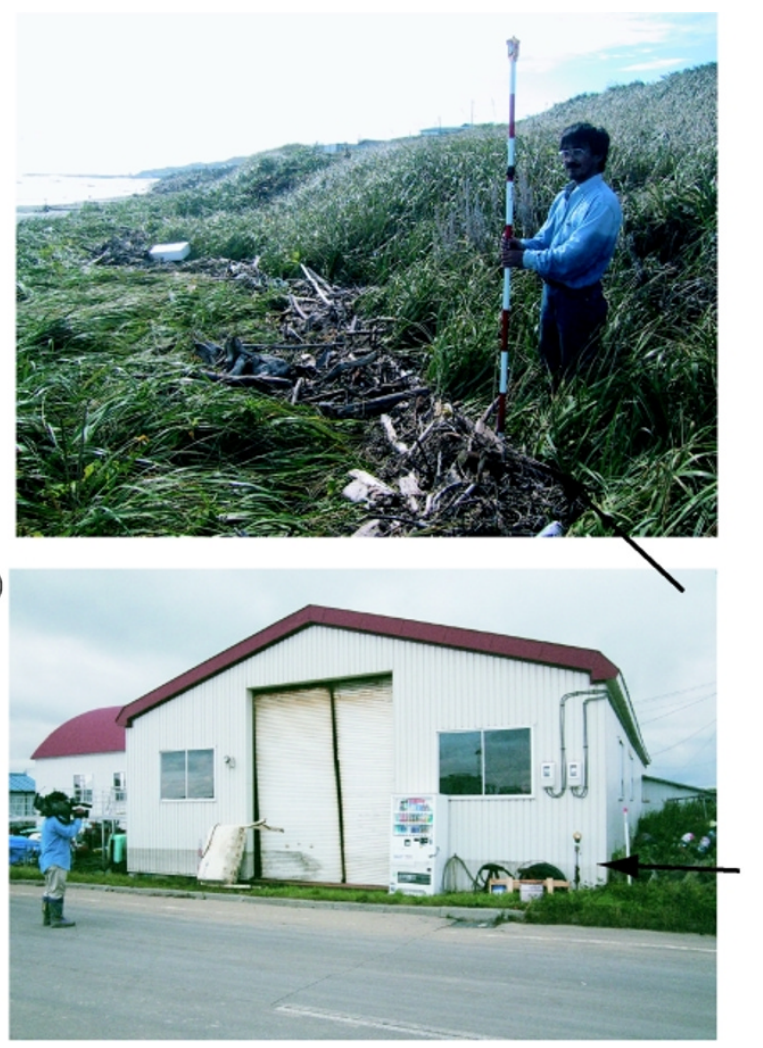

Fig. 2. a) A photo of the tsunami deposits carried by the 2003 tsunami where the tsunami height is measured. b) A photo of the water line around the building which was marked by the inundated tsunami. 


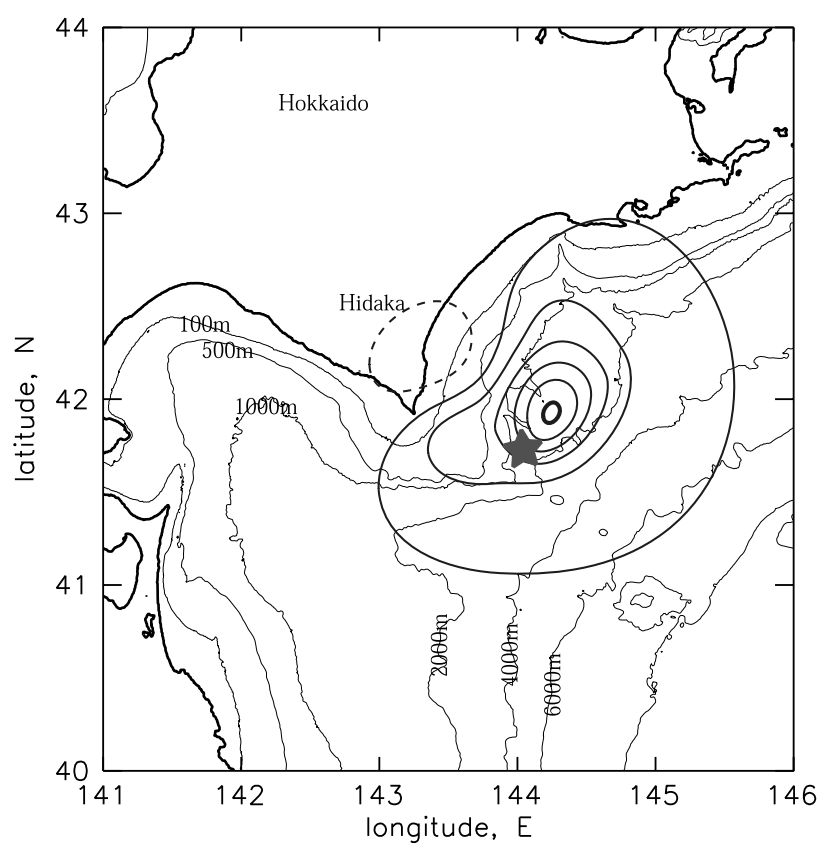

Fig. 3. Vertical deformation due to the 2003 Tokachi-oki earthquake calculated from the slip distribution estimated by Yamanaka and Kikuchi (2003). The star shows the epicenter of the mainshock. Closed contours show the vertical deformation (solid curves for uplift and dashed for subsidence with a $20 \mathrm{~cm}$ interval).

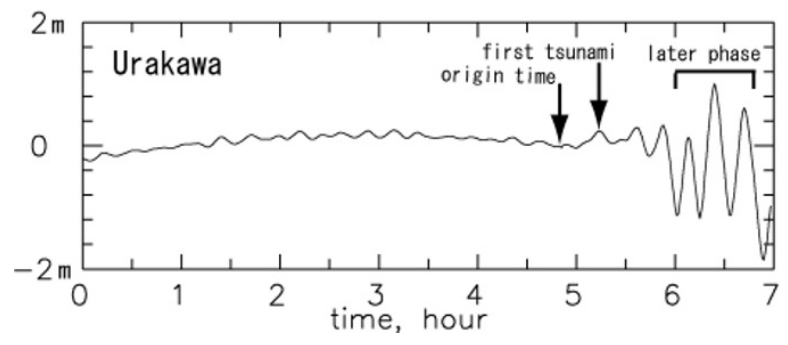

Fig. 4. The tsunami waveform recorded at the tide gauge in Urakawa. Time is the Japan standard time on September 26, 2003.

that the computed tsunamis are too small at Mabiro where a large tsunami was observed locally. Future studies using a nonlinear run-up computation with a finer grid system are necessary to understand the tsunami propagation in these regions.

\section{A Large Later Phase of the Tsunami in the West Coast of Hidaka}

The tsunamis generated by the 2003 Tokachi-oki earthquake were observed at tide gauges in the eastern Hokkaido and the northern Tohoku regions (Hirata et al., 2003). Among them, the tsunami observed at Urakawa shows interesting waveforms (Fig. 4). The largest tsunami arrived about one hour after the first wave, and the amplitude was four times larger than the first wave. Snapshots of the computed tsunamis in Fig. 5 show that the largest tsunami arrived at Urakawa propagated from Cape Erimo along the west coast of the Hidaka area. The first wave at Urakawa was small because the large coseismic deformation area due to the earthquake was located on the other side of Cape Erimo (Fig. 3). The largest tsunami slowly propagates along the coast and

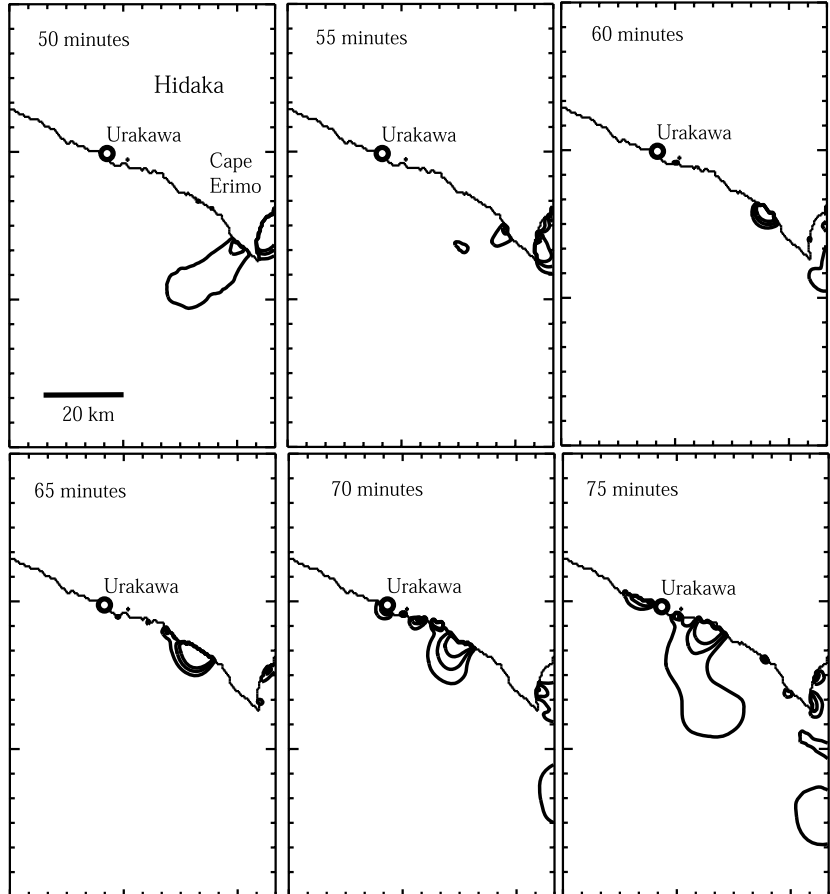

Fig. 5. Six snapshots of the computed tsunami propagation near the west coast of Hidaka area at 50, 55, 60, 65, 70, 75 minutes after the origin time of the earthquake. The contours show the tsunami heights of $15 \mathrm{~cm}, 30$ $\mathrm{cm}, 45 \mathrm{~cm}$.

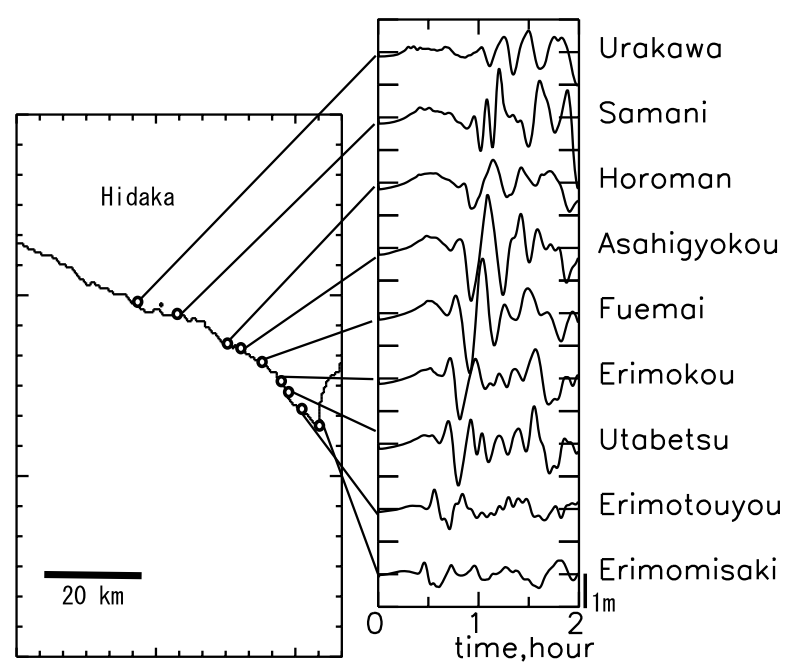

Fig. 6. The computed tsunami waveforms on the west coast of Hidaka Left map shows the location of the stations where the tsunami waveforms are computed. One tick mark in the vertical scale is $1 \mathrm{~m}$. Time is measured from the earthquake origin time.

arrives at Urakawa (Fig. 5). This type of wave is called an edge wave (Gonzalez et al., 1993). Figure 6 shows that the computed tsunami waveforms at the tsunami survey points between Cape Erimo and Urakawa. The later large phases of the tsunami, the edge waves, slowly propagate from Cape Erimo to Urakawa (Fig. 6). In order to find the arrival time of the maximum tsunami at the survey points between Cape Erimo and Urakawa, we used the computed tsunami waveforms in Fig. 6. The arrival times at those survey points are shown in Table 1 and used to correct the tide level. 


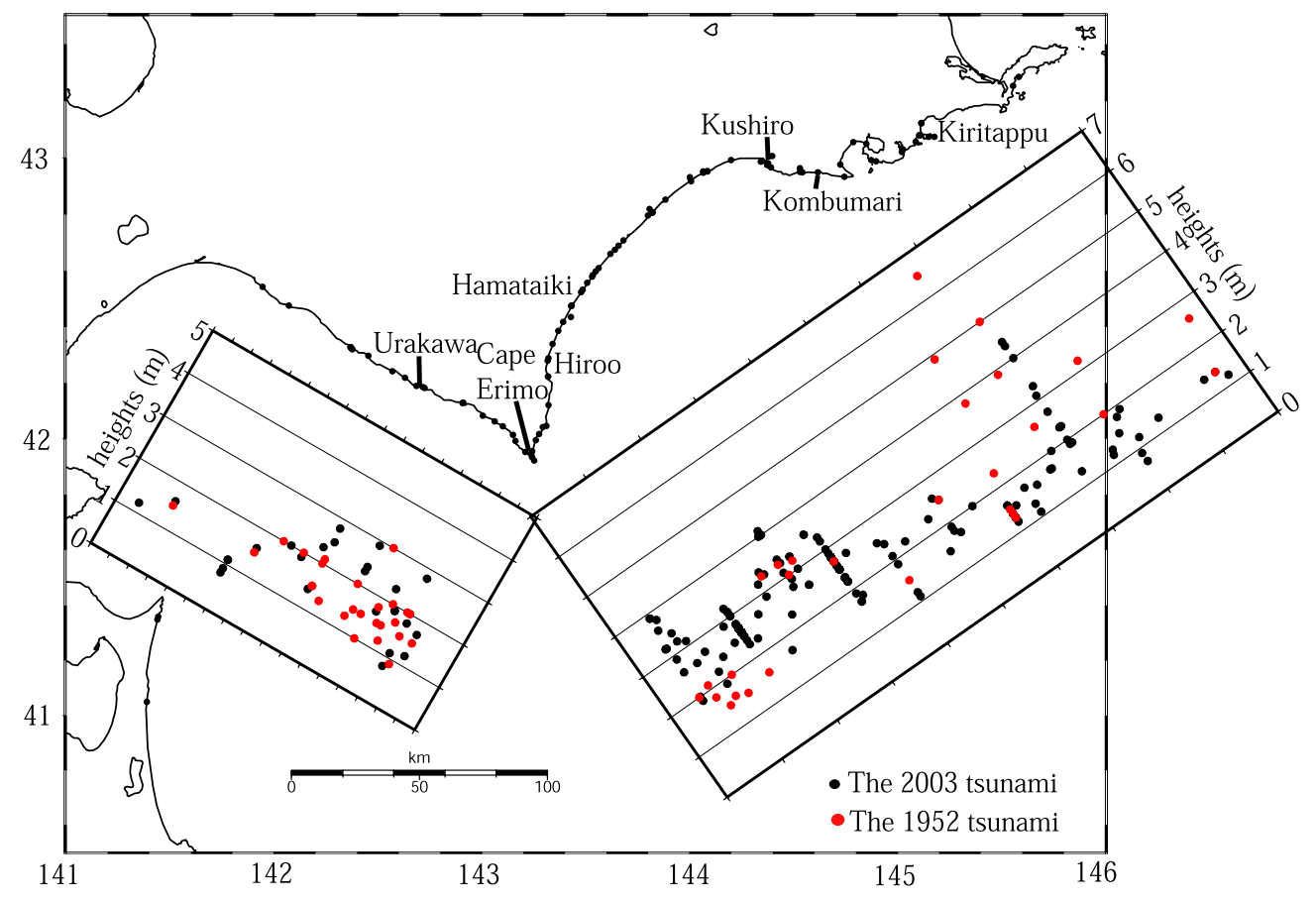

Fig. 7. Comparison of the observed tsunami heights of the 2003 Toklachi-oki earthquake (black circles) and those of the 1952 Tokachi-oki earthquake (red circles) reported by the Central Meteorological Observatory (1953).

\section{Comparison of the Tsunami Heights of the 1952 and 2003 Tokachi-oki Tsunamis}

The tsunami run-up heights of the 1952 Tokachi-oki earthquake were surveyed by the Central Meteorological Observatory (1953). The large tsunami heights of the 1952 Tokachi-oki event, 4-7 m, were observed between Kombumori and Kiritappu (Fig. 7). The tsunami heights of the 2003 event in this area were much smaller than the tsunami heights of the 1952 event (Fig. 7). In the regions between Kushiro and Hamataiki, and west side of Cape Erimo, the tsunami heights of the 1952 and 2003 events are similar to each other. In the region between Cape Erimo and Hiroo, the tsunami heights of the 2003 event were larger than those of the 1952 event. The large differences of tsunami heights in the region between Kombumori and Kiritappu indicate that the source processes or tsunami generation of the 2003 earthquake are different from those of the 1952 earthquake.

\section{Conclusion}

The tsunami survey immediately after the 2003 Tokachioki earthquake revealed that the tsunami height distribution of the 2003 Tokachi-oki earthquake is different from that of the 1952 Tokachi-oki earthquake. The result of the numerical simulation of the 2003 Tokachi-oki tsunami using the slip distribution estimated from the seismological data (Yamanaka and Kikuchi, 2003) shows that the overall pattern of the observed tsunami heights are explained by the computed ones. This indicates that the 2003 Tokachi-oki tsunami is not an unusual tsunami. The differences between the 1952 and 2003 tsunami height distributions suggest that the source processes or tsunami generation of the 1952 earthquake are different from those of the 2003 earthquake. The large later phase observed at the tide gauge in Urakawa is the edge wave propagating from Cape Erimo along the west coast of the Hidaka area.

Acknowledgments. We would like to thank the Japan Meteorological Agency, and the Hydrographic and Oceanographic Department of the Japan Coast Guard for providing us digital tide data. We also thank Kei Katsumata for helping to make figures. The article was significantly improved through the constructive review by Kenji Satake. This work was partially supported by Special Coordination Funds for Promoting Science and Technology, from Ministry of Education, Sports, Culture, Science and Technology.

\section{References}

Gonzalez, I. F., K. Satake, E. F. Boss, and H. O. Mofjeld, Edge wave and non-trapped modes of the 25 April 1992 Cape Mendocino Tsunami, Pure Appl. Geophys., 144, 409-426, 1995.

Hirata, K., Y. Tanioka, K. Satake, S. Yamaki, and E. L. Geist, The tsunami source area of the 2003 Tokachi-oki earthquake estimated from tsunami travel times and its relationship to the 1952 Tokachi-oki earthquake, Earth Planets Space, 56, this issue, 367-372, 2004.

Ichiyanagi, M., H. Takahashi, and M. Kasahara, Aftershock activity of the 2003 Tokachi-oki earthquake $\left(\mathrm{M}_{J M A}=8.0\right)$, Geophysical Bulletin of Hokkaido University, 64, 2004 (in press).

Okada, Y., Surface deformation due to shear and tensile faults in a halfspace, Bull. Seismol. Soc. Am., 75, 1135-1154, 1985.

Satake, K., Linear and nonlinear computations for the 1992 Nicaragua earthquake tsunami, Pure Appl. Geophys., 144, 455-470, 1995.

The Central Meteorological Observatory, Report of the Tokachi-Oki earthquake of March 4, 1952, Quarterly Journal of Seismology, 17, 135 pp., 1953.

Yamanaka, K. and M. Kikuchi, Source processes of the Tokachi-oki earthquake on September 26, 2003, inferred from teleseismic body waves, Earth Planets Space, 55, e21-e24, 2003.

Y. Tanioka (e-mail: tanioka@eos.hokudai.ac.jp), Y. Nishimura, K. Hirakawa, F. Imamura, I. Abe, Y. Abe, K. Shindou, H. Matsutomi, T. Takahashi, K. Imai, K. Harada, Y. Namegawa, Y. Hasegawa, Y. Hayashi, F. Nanayama, T. Kamataki, Y. Kawata, Y. Fukasawa, S. Koshimura, Y. Hada, Y. Azumai, K. Hirata, A. Kamikawa, A. Yoshikawa, T. Shiga, M. Kobayashi, and S. Masaka 